\title{
Parasympathetic functions in children with sensory processing disorder
}

\section{Roseann C. Schaaf ${ }^{1}$, Teal Benevides ${ }^{2}$, Erna Imperatore Blanche ${ }^{3}$, Barbara A. Brett-Green ${ }^{4}$, Janice P. Burke ${ }^{5}$, Ellen S. Cohn' ${ }^{6}$, Jane Koomar', Shelly J. Lane ${ }^{8}$, Lucy Jane Miller', Teresa A. May-Benson,10, Diane Parham ${ }^{11}$, Stacey Reynolds ${ }^{12}$ and Sarah A. Schoen ${ }^{9}$}

1 Department of Occupational Therapy, Jefferson School of Health Professions and Farber Institute for Neuroscience, Thomas Jefferson University, Philadelphia, PA, USA

2 Department of Occupational Therapy, Jefferson School of Health Professions, Thomas Jefferson University, Philadelphia, PA, USA

${ }^{3}$ Division of Occupational Science and Therapy, University of Southern California, Los Angeles, CA, USA

${ }^{4}$ Sensory Processing Disorders Foundation, Greenwood Village, CO, USA

${ }^{5}$ Department of Occupational Therapy, School of Health Professions, Thomas Jefferson University, Philadelphia, PA, USA

${ }^{6}$ Sergeant College of Health and Rehabilitation Sciences, Boston University, Boston, MA, USA

7 Occupational Therapy Associates-Watertown, Watertown, MA, USA

${ }^{8}$ Department of Occupational Therapy, Virginia Commonwealth University, Richmond, VA, USA

${ }^{9}$ Sensory Processing Disorders Foundation, Greenwood Village, CO, USA

${ }^{10}$ The Spiral Foundation, Watertown, MA, USA

"Department of Pediatrics, University of New Mexico, Albuquerque, NM, USA

${ }^{12}$ Virginia Commonwealth University, Richmond, VA, USA

Edited by:

Sidney A. Simon, Duke University, USA

Reviewed by:

Terry Pivik, Arkansas Children's

Nutrition Center, USA

Warren H. Meck, Duke University, USA

${ }^{*}$ Correspondence:

Roseann C. Schaaf, Department of Occupational Therapy, Thomas

Jefferson University, 130 S. 9th Street,

Suite 810, Philade/phia, PA 19107, USA.

e-mail: roseann.schaaf@jefferson.edu
The overall goal of this study was to determine if parasympathetic nervous system (PsNS) activity is a significant biomarker of sensory processing difficulties in children. Several studies have demonstrated that PsNS activity is an important regulator of reactivity in children, and thus, it is of interest to study whether PsNS activity is related to sensory reactivity in children who have a type of condition associated with sensory processing disorders termed sensory modulation dysfunction (SMD). If so, this will have important implications for understanding the mechanisms underlying sensory processing problems of children and for developing intervention strategies to address them. The primary aims of this project were: (1) to evaluate PsNS activity in children with SMD compared to typically developing (TYP) children, and (2) to determine if PsNS activity is a significant predictor of sensory behaviors and adaptive functions among children with SMD. We examine PsNS activity during the Sensory Challenge Protocol; which includes baseline, the administration of eight sequential stimuli in five sensory domains, recovery, and also evaluate response to a prolonged auditory stimulus. As a secondary aim we examined whether subgroups of children with specific physiological and behavioral sensory reactivity profiles can be identified. Results indicate that as a total group the children with severe SMD demonstrated a trend for low baseline PsNS activity, compared to TYP children, suggesting this may be a biomarker for SMD. In addition, children with SMD as a total group demonstrated significantly poorer adaptive behavior in the communication and daily living subdomains and in the overall Adaptive Behavior Composite of the Vineland than TYP children. Using latent class analysis, the subjects were grouped by severity and the severe SMD group had significantly lower PsNS activity at baseline, tones and prolonged auditory. These results provide preliminary evidence that children who demonstrate severe SMD may have physiological activity that is different from children without SMD, and that these physiological and behavioral manifestations of SMD may affect a child's ability to engage in everyday social, communication, and daily living skills.

Keywords: parasympathetic nervous system, sensory processing disorder, modulation, vagal tone, heart period variability, respiratory sinus arrhythmia

\section{INTRODUCTION}

Approximately $5-10 \%$ of the non-disabled population and $30 \%$ of children with disabilities experience atypical responses to sensory stimuli that interfere with their ability to fully participate in home, school, and community activities (Baranek, 1998; Ahn et al., 2004). In this paper, we use the term sensory modulation dysfunction (SMD) to refer to patterns of atypical responses to typical levels of sensation Lane (Lane, 2002). SMD is a proposed subtype of larger problem sometimes termed "Sensory Processing Disorder" or SPD (Miller et al., 2007).SPD is thought to occur when sensory information is perceived and interpreted in a disorganized way so that the persons ability to use sensory information to act and interact with their environment is impaired (Miller, 2006). This disorder was originally described and researched by Ayres $(1972,1979)$. Ayres 
(1972) developed the theory of sensory integration to describe the neurobiological process of organizing sensations from one's own body and the environment for effective interactions within the environment. Clinical descriptions of people with SMD indicate that they misinterpret everyday sensory information, such as touch, sound, and movement; they may feel bombarded by information, seek out intense sensory experiences, or fail to notice and respond to sensory information that typically captures attention (Miller, 2006). This creates challenges for them in their everyday tasks as they may have difficulty paying attention, are at risk for learning and behavioral difficulties and social isolation, or may be disturbed or distressed by typical levels of sensation, such as the sound of a toilet flushing or tolerating the way socks feel on one's feet. Although it is clear that SMD is a significant factor that limits participation in everyday tasks, the physiological mechanisms involved in SMD are not well understood, nor are the mechanisms by which various therapeutic approaches for these difficulties are thought to work. As a result, data supporting interventions that address underlying mechanisms of SMD are sparse and lack evidence to support their efficacy (Miller, 2003). This study addresses this gap by determining if atypical parasympathetic nervous system (PsNS) activity is a significant physiological factor in SMD. These data will provide a more complete understanding of the mechanisms of SMD and provide the foundation for future studies that will examine whether therapeutic interventions that target the PsNS are effective for helping children with sensory difficulties participate more successfully in their environments.

The literature associated with exploring the nature of sensory processing problems has primarily addressed behavioral patterns using psychometric and multivariate methods, such as factor analysis, to identify categories of dysfunction (Ayres, 1969, 1989; Dunn, 1999; Bundy et al., 2002). While the study of sensory processing problems from a behavioral perspective has been useful in providing clinicians with data to guide practice, it has not provided insight into the underlying physiological mechanisms of SMD, or the potential implications of these for the development of intervention strategies. One notable exception is the work of Miller and colleagues (McIntosh et al., 1999a,b; Miller et al., 1999). Using their unique paradigm, the Sensory Challenge Protocol (SCP), they measured electrodermal reactivity, a marker of sympathetic nervous system (SNS) activity, during the administration of sensory stimuli (McIntosh et al., 1999b; Miller et al., 2001). The findings from these studies provided initial evidence that children with SMD demonstrate unique, atypical patterns of sympathetic activity, and have laid the foundation for differentiating SMD as a distinct set of conditions based on physiological and behavioral characteristics. Miller and her colleagues note the need for further study of the ANS correlates of SMD, especially studies that include role of the PsNS (Miller et al., 1999).

The autonomic nervous system regulates an individual's ability to adapt to environmental changes through modulation of sensory, motor, visceral, and neuro-endocrine functions via its parasympathetic and sympathetic branches. These branches function together to promote adaptation and self-regulation in response to internal and external environmental demands. The sympathetic branch of the autonomic nervous system modulates immediate phasic responses to events, such as the fight-or-flight reaction, while the parasympathetic branch modulates the visceral and the neuro-endocrine systems to maintain homeostasis and selfregulation, as well as to regulate recovery from a stressor/challenge (Nance and Hoy, 1996). Given that children with SMD demonstrate severe over- or under-responsiveness to sensation, often with inability to restore homeostasis or self-regulation after a stressor or challenge, it is reasonable to suspect that they may have disturbances in autonomic nervous system functions that influence their ability to participate in everyday activities (McIntosh et al., 1999b). The study of PsNS activity in individuals with SMD will contribute to our understanding of sensory processing problems, and will provide data that will be used in future studies to design and test interventions to help individuals affected by SMD.

As noted above, investigation of the role of the PsNS in SMD is very limited (Schaaf et al., 2003). Research on children with other disabilities, however, suggests that parasympathetic function may be a useful index of self-regulation (Fox and Porges, 1985; Malliani, 1995; Porges, 1995; Boccia and Roberts, 2000). High parasympathetic activity, measured by assessing heart rate variability in the high frequency band of respiration has been associated with homeostasis and with the ability to adaptively cope with a wide range of changing stimuli. Decreased and disorganized parasympathetic activity, on the other hand, is associated with a narrow range of behavioral adaptation to changing stimuli, and is a predictor of stress, vulnerability, and risk status (DiPietro et al., 1987; DeGangi et al., 1991; Porges and Byrne, 1992; Malliani, 1995; Porges, 1995). Boccia and Roberts (2000) found that boys with fragile X syndrome, who are extremely hyper-responsive to sensation, had decreased PsNS activity. Similarly, Huffman et al. (1998) showed that infants with poor behavioral adaptability also demonstrated low PsNS activity. Further, the literature suggests that when the PsNS is not effectively regulating the child's responses to stimuli, the sympathetic nervous system response is also impaired (Cacioppo et al., 1994; Porges, 1995; Beauchaine, 2001; Berntson et al., 2008). Data from these studies suggest a need to examine PsNS functioning in children with SMD who by definition have unusual responses to stimuli, often accompanied by limited behavioral flexibility and variable clinical outcomes.

Porges' $(1995,2007)$ Polyvagal Theory describes the potential relationship of PsNS activity to behavioral adaptability. Application of this theory to children with SMD suggests that these children may have aberrant PsNS activity that underlies their sensory dysfunction, and, therefore, they respond to environmental challenges and stimuli with ineffective strategies for maintaining behavioral regulation. Since they are unable to engage PsNS responses to cope with the typical levels of stimuli in the environment in flexible and adaptive ways, they demonstrate ineffective and atypical behavioral responses to sensation. It is hypothesized that the PsNS does not work in an adaptive manner to help regulate responses to stimuli and thus, PsNS responses during sensory challenge are disorganized, resulting in atypical behavioral responses that interfere with the individual's participation in daily tasks.

In this study, the term "baseline vagal tone" will be used to describe the participant's resting level of PsNS activity as measured by the variability in heart period in the high frequency band of respiration. Vagal tone (VT) is also referred to in the literature by the term high frequency heart rate variability (Chambers 
and Allen, 2007) or respiratory sinus arrhythmia (Berntson et al., 1997) and is frequently used in the literature as a non-invasive marker of PsNS activity and as a marker of behavioral regulation (Porges, 1981, 1985; Fox and Stifter, 1989). The decrease in VT from baseline to challenge, called "vagal suppression" or "vagal reactivity", is used in the literature to assess the dynamic reactivity of the vagal system. Porges (1995) and Porges et al. (1996) found that suppression of VT during a stressor may function as an adaptive response to increase the individual's orientation to external stimuli thereby allowing regulation and coordination of other complex responses to stimuli (i.e., sympathetic or adrenal response). Similarly, Calkins and Keane (2004) showed that specific patterns of vagal change from baseline to stimuli are associated with different temperament and coping styles. In order to examine vagal reactivity, the difference between baseline VT and VT during sensory challenges was used to describe the participant's ability to cope with the sensory stimuli. Finally, "vagal recovery", defined by a child's average cardiac VT during a 3-min recovery period, is used to describe the participant's return to parasympathetic control following a sensory challenge, and therefore their ability to adapt to the environment.

The specific research questions addressed by this study are:

- What differences in baseline VT, vagal reactivity during sensory challenge, and vagal recovery exist between children with SMD compared to typically developing (TYP) children with no sensory dysfunction during a controlled laboratory procedure?;

- What patterns of vagal reactivity occur within the group of children with SMD compared to the TYP children?; and

- Does PsNS activity predict atypical sensory behaviors and adaptive functions in children with SMD in comparison to TYP controls?

As a secondary analysis, we also examined whether there is evidence for subgroups of children with SMD with specific physiological and behavioral profiles.

Based on the literature reviewed above, the following hypotheses were proposed:

1. Children with SMD will show significantly lower baseline VT and lower VT during sensory challenge than TYP children without sensory or other diagnoses.

2. Children with SMD will demonstrate different vagal reactivity to sensory challenge than TYP controls.

3. Low baseline VT and low vagal reactivity will be associated with higher frequency of atypical sensory behaviors and poorer adaptive behaviors as predicted by Porges' Polyvagal theory.

4. Children with SMD can be sub-grouped according to behavioral responses to sensation that predict differential vagal activity during sensory challenge.

\section{MATERIALS AND METHODS PARTICIPANTS}

A total of 83 subjects ( 40 TYP children and 43 SMD children), ages 5-12 years, were included in the study. Approval to conduct the study was gained from the Institutional Review Board at Thomas Jefferson University (TJU) of Philadelphia, PA. Approval was granted via an Interagency Authorization Agreement to conduct the study at Pediatric Therapy Network by the TJU IRB. Research participants at both sites completed parental permission and child assent (for children ages 7 and older) according to approved procedures. The TYP sample included 18 male (45\%) and 22 female (55\%) children. The SMD sample included 29 male $(71 \%)$ and 12 female $(29 \%)$ and 2 with unknown gender. Children who were missing age data were excluded from some of the analyses, thus participant numbers may vary. Parental demographic socioeconomic status (SES) was reported using our demographics questionnaire which is based on Hollingshead Two Factor Index of Social Position (Hollingshead, 1991). Education level for the primary parent (parent who self identified themselves with main role of caring for the child's day to day activities, and who served as the primary respondent in our study) was not significantly different between the total TYP and SMD samples $\left(\chi^{2}=3.12, p=0.37\right)$. Of the parents who completed the study with their child, $5.6 \%$ had a high school degree or less, $11.1 \%$ had a 2 -year college degree or equivalent, $47.2 \%$ had a 4 -year college degree or equivalent, and $36.1 \%$ of parents had completed graduate education.

As per our approved procedures, subjects are permitted to request to stop or skip testing. Of the subjects with SMD, four asked to skip a sensory domain or stop testing. An additional seven SMD children had unusable physiological data due to movement artifacts or equipment malfunction. One TYP child (3\%) asked to skip a sensory domain, and two TYP children (5\%) have missing data due to equipment malfunction. These children were not included in the analyses.

All children met the following inclusion criteria for the study: (1) between the ages of 5 and 12 years of age; (2) no physical or medical complications that affected cardiac activity; (3) no history of seizure activity; (4) no medications that affected cardiac activity (children were excluded if they were medications that affected autonomic tone or cardiac activity including betablockers, adderall, ditropan, or SSRI's); (5) must be able to follow simple directions, sit with the examiner during the testing, and tolerate electrode placement. Children with SMD had to meet additional criteria: (1) referral for atypical sensory responsivity made by clinician, pediatrician, neurologist or self-referral; and (2) meet the criteria for SMD based on one of the following scores on the Short Sensory Profile (SSP): (a) Total Test score of less than 3.0 SD below the mean; (b) less than $2.5 \mathrm{SD}$ below the mean on two or more subtests; or (c) less than 4.0 SD below the mean on one subtest (Miller et al., 2001).

\section{PROCEDURES}

The children and parents in this study came from populations at Thomas Jefferson University, in Philadelphia, PA, and from Pediatric Therapy Network in Torrance, CA. All subjects were tested by author Teal Benevides at their respective sites (PA or CA). Following a phone screening to determine eligibility, parents scheduled a 1.5 -h visit with their child in which they underwent informed consent and child assent procedures, the child was introduced to the equipment and electrodes used to measure ECG and sweat responses, and the child completed a 35-min physiological session called the SCP (described below). The primary parent completed questionnaires regarding their child's sensory dysfunction, 
adaptive behavior, and relevant demographic questions. Parents were invited to observe their child's physiological testing inconspicuously by watching from the back of the room or from a twoway mirror.

\section{MEASURES}

\section{The short sensory profile}

The SSP (Miller et al., 1999) was used to screen for and assess sensory responsivity. The SSP is a 38 -item parent rating scale that examines sensory responsivity (tactile sensitivity, taste/smell sensitivity, movement sensitivity, and visual/auditory sensitivity, auditory filtering, sensation seeking, and measures proprioception or the ability to use muscles to move in daily life) and the impact of these on daily life activities. It was developed as a research tool from the longer Sensory Profile (Dunn, 1999). The instrument has strong internal reliability $(>0.80)$ and construct validity, and takes about $20 \mathrm{~min}$ for the parent or guardian to complete.

\section{The vineland adaptive behavior scales II}

The Vineland Adaptive Behavior Scales II (VABS-II; Sparrow et al., 2005) was used to assess adaptive behaviors needed for participation in home, school and community activities. The VABS-II is a standardized, norm-referenced measure that determines adaptive behavior in four domains: Communication Skills (Receptive, Expressive, Written), Daily Living Skills (Personal, Domestic, Community), Socialization Skills (Interpersonal Relationships, Play and Leisure, Coping), and a Motor Skills domain for children under the age of 6 years 11 months. The VABS-II has strong psychometric properties and is used in the literature as an index of adaptive behavior.

\section{The sensory challenge protocol}

Physiological responses were measured during the SCP, a unique research protocol designed to measure ANS activity in a controlled laboratory environment. The laboratory is designed to be fun and interesting for the child and is decorated to look like a space ship with stars and planets. The SCP consists of four phases: (1) baseline period of 3 min while the child is seated quietly in a chair; (2) administration of sensory challenges (seven stimuli repeated consecutively eight times each), (3) recovery phase where the child returns to quiet sitting for $3 \mathrm{~min}$, and (4) a 2-min auditory tone (prolonged stimulus delivered after recovery. The stimuli include: auditory tones (at 84 decibels), visual (20 W strobe light at $10 \mathrm{~Hz}$ ), auditory siren sound (at 78 decibels), olfactory (wintergreen oil passed under the nose), tactile (touch along the jaw bone from the mandibular angle on the right to the mandibular angle on the left with a feather), and vestibular (chair tilted back to a $30^{\circ}$ angle). A 2-min prolonged auditory tone (75 decibels) was administered after recovery ${ }^{1}$. All stimuli but the prolonged auditory stimuli occur for $3 \mathrm{~s}$ with a pseudo-random interval of 12-17 s between stimuli

\footnotetext{
${ }^{1}$ The prolonged auditory was administered after recovery. The purpose of this stimuli is to assess vagal response (i.e., regulation) over a longer period of time as opposed to vagal response to shorter duration stimuli. Administration of prolonged auditory after recovery was necessary because we are sharing data with additional laboratories for other studies. These labs do not include the prolonged auditory stimuli and thus, including it at the end of the SCP assured that shared data would be similar.
}

and approximately 30-s interval between sensory domains. The examiner is present during data collection and records the child's behavioral responses to the sensory challenge, as well as administers tactile and olfactory sensations. Prior to the administration of each sensory modality the child is given the instruction "now you are going to hear/feel/see something." Little to no interaction occurs between the experimenter and child during the administration of stimuli. Physiological data are collected continuously during the experiment. The SCP has been used successfully with clinical populations in studies by McIntosh et al. (1999b) and Schaaf et al. (2003).

Electrocardiogram data were gathered during the SCP to obtain an index of parasympathetic function. The VT index, the marker of PsNS activity, was compared for each group during each phase of the SCP: baseline, each sensory challenge, recovery, and prolonged auditory phases. The VT is calculated by assessing the variability of inter-beat intervals of the electrocardiogram, also called heart period, within the high frequency band of respiration. To obtain the VT, raw ECG data were collected using PsyLab SAM (Contact Precision Instruments, 2002), a psychophysiological data logger. Three Ag/AgCl electrodes were placed in a triangle configuration on the child's chest, with two leads placed near the axilla approximately level with the heart, and a ground lead placed midline on the child's stomach. Raw heart rate data were sampled at $1000 \mathrm{~Hz}$, and R-R interval obtained with a wave-shape recognition algorithm, accurate to $1 \mathrm{~ms}$. The resulting train of IBI data is sampled beat by beat in the VT analysis process. PSYLAB software (Contact Precision Instruments, 2002) was used to reduce the ECG data into heart period data which was then imported into MXEdit (Delta Biometrics, 1993), a software program specifically designed to allow for accurate editing of artifacts in the data, and evaluation of the variability of heart period within the high frequency range of respiration through use of a moving polynomial filter. Artifact identification and editing follows the procedures and guidelines delineated in the MXEdit training manual, and involves the identification of outlier points and the incorporation of outlier data points into the existing heart period pattern via summation or division. Authors RS and TB completed MXEdit training and reliability certification. Data files that required more than $10 \%$ of the data points to be edited were not included in the analyses. Following editing, the VT was calculated using the method patented by Porges (1980). A 21-point moving polynomial filter is used to detrend the heart period data within 30-s epochs, and determine the high frequency variance associated with this detrended data. For our sample of children, a high frequency band of 0.25-1.04 Hz was used (Calkins and Keane, 2004). Cardiac VT, as referred to in this article, is the natural logarithm of heart period variability within the high frequency band of respiration, and is reported in $\log (\mathrm{ms})^{2}$. Average cardiac VT over each 30-s epoch was used to describe a child's parasympathetic response during baseline, each domain of the SCP, recovery, and prolonged auditory stimuli.

\section{STATISTICAL ANALYSES}

A quasi-experimental design was used to evaluate differences in PsNS functions in children with SMD compared to a TYP control group. Mixed effects linear regression was used to model 
VT measurements. Fixed effects were included for VT domain (baseline, tones, visual, siren, olfactory, tactile, vestibular, recovery, prolonged auditory), diagnosis group (TYP or SMD), and their interaction. A fixed effect was also included for chronological age to control for this variable. A random intercept term was included to account for correlation among repeated measurements from the same subject.

Within this model, we tested for differences between groups at baseline and each sensory domain, recovery and prolonged auditory challenge. We also tested for difference in change from baseline to each of the sensory challenges, and change from baseline to recovery for each group. Next, we performed a latent class analysis using SSP scores to determine if there were meaningful subgroups in the SMD children and then compared difference between SMD groups and TYP at baseline and at each domain of the SCP; and tested for difference in change from baseline to each sensory challenge in these subgroups. We treated domain-specific tests as tests of separate hypotheses, each with a type-I error rate of $5 \%$. For the comparison of latent classes to control, we used a Bonferroni-adjusted type-I error rate of $1.6 \%$ (5\%/3 comparisons per domain).

\section{RESULTS}

\section{DIFFERENCES IN MEAN VAGAL TONE BETWEEN SMD AND TYP SAMPLES}

For these analyses 79 subjects were included (38 TYP and 41 SMD) as four subjects without age data were excluded. As hypothesized, the SMD subjects as a total group had lower mean baseline VT than the TYP subjects, and this approached significance in the hypothesized direction $(\mathrm{SMD}=6.08$; TYP $=6.55 ; p=0.083)$. In addition, as hypothesized, children with SMD overall had lower mean VT than TYP children at each sensory challenge domain, but these findings were non-significant. There were no significant differences between groups on mean VT during recovery or prolonged auditory stimuli. Of note, the SMD group returned to baseline level of VT at recovery whereas the TYP group shows lower VT at recovery compared to baseline. Both groups return to baseline level of VT during the prolonged auditory stimuli. Mean VT for SMD and TYP groups are displayed in Figure 1, and mean values, confidence intervals and $p$ values between group comparisons are listed in Table 1.

\section{VAGAL REACTIVITY IN CHILDREN WITH SMD COMPARED TO TYP SAMPLE}

Vagal reactivity was defined as the change in VT from baseline to each sensory challenge domain. The ability to suppress VT in response to a challenge is reported as a negative change, and an increase in VT in response to a challenge is reported as a positive change (Challenge - BL level). Within group vagal change from baseline to challenge domain was significant for visual, siren, tactile and vestibular domains for the SMD group and for olfactory and vestibular for the TYP group as shown in Table 2. In addition, significant differences between group changes in vagal reactivity were noted during the visual, siren, olfactory, tactile and vestibular domains. It is interesting to note that in general, the SMD group increased VT from baseline to these stimuli whereas the TYP group showed minimal reactivity or a decrease (olfactory). One exception is vestibular reactivity where both groups demonstrated increased vagal reactivity, but the SMD

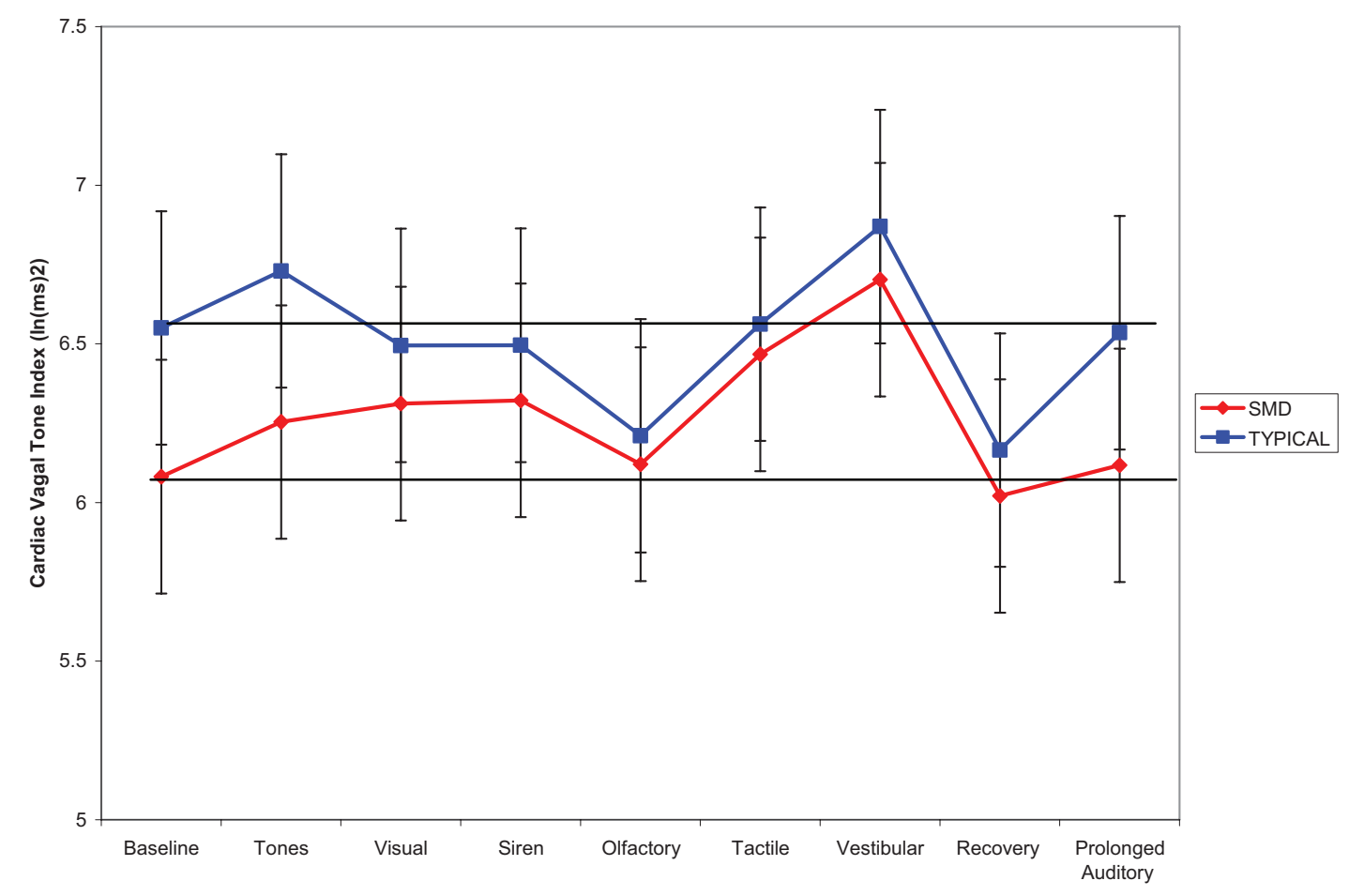

FIGURE 1 | Mean VT during Sensory Challenge Protocol for SMD versus TYP. (Horizontal line drawn to indicate baseline level for each group). 
group increased their VT significantly more than the TYP. Of note, the vestibular stimulus is multisensory in nature in that the chair makes noise as it moves and vibrates slightly. A significant difference between SMD and TYP groups for the change from baseline to recovery was found. The SMD group returned to baseline level of VT during recovery, but the TYP group demonstrated significantly

\begin{tabular}{llll}
$\begin{array}{l}\text { Table 1 | Age-adjusted mean VT values for SMD and TYP groups } \\
\text { during SCP. }\end{array}$ & & \\
\hline SCP domain & SMD mean & TYP mean & Between group \\
& VT (95\% Cl) & VT (95\% Cl) & difference $\boldsymbol{p}$ value \\
\hline Baseline & $6.08(5.71-6.45)$ & $6.55(6.18-6.92)$ & 0.083 \\
Tones & $6.25(5.88-6.62)$ & $6.73(6.36-7.10)$ & 0.080 \\
Visual & $6.31(5.94-6.68)$ & $6.50(6.13-6.86)$ & 0.50 \\
Siren & $6.32(5.95-6.69)$ & $6.50(6.13-6.87)$ & 0.52 \\
Olfactory & $6.12(5.75-6.49)$ & $6.21(5.84-6.58)$ & 0.74 \\
Tactile & $6.47(6.10-6.83)$ & $6.56(6.19-6.93)$ & 0.73 \\
Vestibular & $6.70(6.33-7.07)$ & $6.87(6.50-7.24)$ & 0.54 \\
Recovery & $6.02(5.65-6.39)$ & $6.17(5.80-6.53)$ & 0.59 \\
Prolonged & $6.12(5.75-6.49)$ & $6.54(0.15-6.91)$ & 0.13 \\
auditory & & &
\end{tabular}

Table 2 | Difference in vagal reactivity between SMD and TYP change score $(95 \% \mathrm{Cl})$.

\begin{tabular}{|c|c|c|c|}
\hline & SMD & TYP & \\
\hline $\begin{array}{l}\text { Change } \\
\text { from BL to } \\
\text { SCP domain }\end{array}$ & $\begin{array}{l}\text { Mean VT change } \\
(95 \% \mathrm{Cl})\end{array}$ & $\begin{array}{l}\text { Mean VT change } \\
(95 \% \mathrm{CI})\end{array}$ & $\begin{array}{l}\text { Difference } \\
\text { between } \\
\text { groups } \\
p \text { value }\end{array}$ \\
\hline BL to tones & $0.17(-0.00$ to 0.37$)$ & $0.18(-0.008$ to 0.36$)$ & ns \\
\hline $\mathrm{BL}$ to visual & $0.23^{*}(-0.04$ to 0.42$)$ & $-0.06(-0.24$ to 0.13$)$ & $0.034^{*}$ \\
\hline BL to siren & $0.24^{*}(0.05-0.43)$ & $-0.05(-0.24$ to 0.13$)$ & $0.030^{*}$ \\
\hline BL to olfactory & $0.04(-0.15$ to 0.23$)$ & $-0.34^{* *}(-0.53$ to -0.15$)$ & $0.005^{* *}$ \\
\hline BL to tactile & $0.39 *(0.20-0.57)$ & $0.01(-0.17$ to 2.0$)$ & $0.006^{* *}$ \\
\hline $\mathrm{BL}$ to vestibular & $0.62 *(0.43-0.81)$ & $0.32 *(0.13-0.50)$ & $0.027^{*}$ \\
\hline$B L$ to recovery & $0.06(0.25-0.13)$ & $-0.39 * *(-0.57$ to 1.20$)$ & $0.017^{*}$ \\
\hline BL to ProAud & 0.04 (-0.16 to 0.23$)$ & $-0.01(-0.23$ to 0.19$)$ & 0.73 \\
\hline
\end{tabular}

Cl, confidence interval; SCP, Sensory Challenge Protocol; BL, baseline; ProAud, prolonged auditory stimuli.

${ }^{*} p<0.05$ level, ${ }^{* *} p<0.01$ level. lower recovery VT than in baseline. Lastly, vagal reactivity between baseline and prolonged auditory was examined between groups and no significant differences were found.

\section{SUBGROUPS OF CHILDREN WITH SMD}

Based on Miller et al.ss findings (Miller et al., 2003), we anticipated that the subjects with SMD would be somewhat heterogeneous in their responses to sensory stimuli and thus, to examine the differences in VT between the SMD group and TYP in more depth, we performed a latent class analysis to determine the best grouping strategy for children with SMD. For this analysis 39 SMD subjects were included (subjects with missing age data were excluded). The best statistical fit for the SMD group was a three class latent class model that grouped subjects by severity of sensory dysfunction based on mean SSP domain scores (Table 3). It should be noted that Miller grouped subjects by type (over-responsive, under-responsive and seeking) using cluster analysis methodology whereas our data grouped best by severity. Latent class $1(n=15)$ was the most severe group, in which children demonstrated lower than 4.0 standard deviations below mean on tactile sensitivity, taste/smell sensitivity, and auditory filtering, and demonstrated 3.0 standard deviations below the mean on under-responsive/seeks sensation and low energy/weak. This class was labeled the "severe SMD" group. Latent class $2(n=13)$ was not as severe in their behavioral responses, demonstrating on average three standard deviations below the mean on tactile sensitivity, auditory filtering, and low energy/weak. Latent class 2 was labeled the "moderate SMD" group. Latent class $3(n=11)$ demonstrated on average 2 standard deviations below the mean on the under-responsive/seeks sensation subdomain only. All other domains were within the typical range for this class of SMD subjects. This group was labeled the "borderline SMD" group.

\section{Differences in mean VT between latent class groups of SMD versus TYP}

Next we examined differences in mean VT during each phase of the SCP for each SMD group compared to TYP. For this analysis, 77 subjects were included (39 TYP and 38 SMD) (subjects with missing age data were excluded). Age-adjusted mean VT for each SMD group and TYP group during each phase of the SCP are reported in Table 4. The severe SMD group (latent class 1) demonstrated lower mean VT than the TYP children for baseline $(p=0.026)$; tones $(p=0.020)$, and prolonged auditory stimuli $(p=0.035)$ which approached significance using the adjusted alpha level (alpha $=0.016)$. There were no significant differences between

Table 3 | Latent class analysis: mean SSP z-scores (95\% Cl) by SMD class.

\begin{tabular}{lllc}
\hline SSP domain & Class $\mathbf{1}(\boldsymbol{n}=\mathbf{1 5})$, severe SMD & Class $\mathbf{2}$ ( $\boldsymbol{n}=\mathbf{1 3})$, moderate SMD & Class $\mathbf{3}(\boldsymbol{n}=\mathbf{1 1}), \mathbf{b o r d e r l i n e ~ S M D ~}$ \\
\hline Tactile sensitivity & $-4.5(-5.2$ to -3.8$)$ & $-3.1(-3.6$ to -2.6$)$ & $-0.5(-1.3$ to 0.4$)$ \\
Taste/smell sensitivity & $-4.8(-5.3$ to -4.3$)$ & $-0.1(-0.9$ to 0.8$)$ & $0.6(-0.7$ to 1.9$)$ \\
Movement sensitivity & $-1.9(-2.8$ to -0.9$)$ & $-0.8(-1.3$ to -0.3$)$ & $0.2(-0.2$ to 0.5$)$ \\
Under-responsive/seeks sensation & $-3.5(-4.4$ to -2.5$)$ & $-2.5(-3.2$ to -1.8$)$ & $-2.0(-3.0$ to -0.9$)$ \\
Auditory filtering & $-4.0(-4.6$ to -3.5$)$ & $-3.4(-4.1$ to -2.6$)$ & $-1.6(-2.5$ to -0.8$)$ \\
Low energy/weak & $-3.5(-4.6$ to -2.3$)$ & $-3.1(-4.1$ to -2.2$)$ & $-0.6(-1.4$ to 0.3$)$ \\
Visual/auditory sensitivity & $-2.8(-3.6$ to -1.9$)$ & $-2.6(-3.3$ to -2.0$)$ & $-0.6(-1.2$ to -0.03$)$
\end{tabular}


the SMD class 2 (moderate SMD) and SMD class 3 (borderline SMD) compared to TYP controls. These results are displayed in Figure 2.

\section{Vagal Reactivity in SMD Subgroups compared to TYP sample}

Vagal reactivity, defined as change in VT from baseline to sensory challenge, recovery and prolonged auditory, was compared between SMD subgroups (severe, moderate, borderline) and TYP children to determine if there were significant within and between group differences. The mean change scores and confidence intervals are

Table 4 | Differences in mean VT values for SMD classes versus TYP groups during SCP.

Age adjusted mean VT $(95 \% \mathrm{Cl})$

SCP SMD group 1, SMD group 2, SMD group 3, TYP mean

domain severe $(95 \% \mathrm{Cl})$ moderate borderline VT $(95 \% \mathrm{Cl})$

\begin{tabular}{lllll} 
& $\mathbf{9 5 \%} \mathbf{~ C l}$ & $\mathbf{9 5 \%} \mathbf{~ C l})$ & $\mathbf{9 5 \%} \mathbf{~ C l})$ & \\
\hline Baseline & $5.81(5.3-6.4)$ & $6.44(5.9-7.0)$ & $6.5(5.8-7.2)$ & $6.6(6.2-6.9)$ \\
Tones & $5.95(5.4-6.5)$ & $6.5(5.9-7.1)$ & $6.9(6.2-7.6)$ & $6.7(6.4-7.1)$ \\
Visual & $6.2(5.7-6.8)$ & $6.6(6.1-7.2)$ & $6.5(5.8-7.2)$ & $6.5(6.1-6.9)$ \\
Siren & $6.2(5.7-6.8)$ & $6.6(6.0-7.2)$ & $6.5(5.8-7.2)$ & $6.5(6.2-6.9)$ \\
Olfactory & $5.8(5.2-6.4)$ & $6.5(5.9-7.1)$ & $6.5(5.8-7.2)$ & $6.2(5.9-6.6)$ \\
Tactile & $6.2(5.6-6.7)$ & $6.6(6.2-7.3)$ & $6.9(6.3-7.6)$ & $6.6(6.2-6.9)$ \\
Vestibular & $6.5(5.9-7.1)$ & $7.2(6.6-7.7)$ & $6.9(6.2-7.6)$ & $6.9(6.5-7.2)$ \\
Recovery & $5.9(5.3-6.5)$ & $6.4(5.8-7.0)$ & $6.1(5.4-6.8)$ & $6.2(5.8-6.5)$ \\
ProAud & $5.8(5.3-6.4)$ & $6.5(5.9-7.0)$ & $6.6(5.9-7.3)$ & $6.6(6.2-6.9)$ \\
\hline
\end{tabular}

$\mathrm{Cl}$, confidence interval; SCP, Sensory Challenge Protocol; BL, baseline; ProAud, prolonged auditory stimuli. reported in Table 5. Significant between group differences existed primarily for the severe SMD group compared to the TYP group for BL to visual $(p=0.01), \mathrm{BL}$ to siren $(p=0.009)$ and BL to recovery $(p=0.002)$. Between group differences for severe SMD compared to TYP approached significance for BL to olfactory $(p=0.07)$, BL to vestibular $(p=0.05)$, and BL to tactile $(p=0.07)$.

\section{ADAPTIVE BEHAVIOR}

Differences between SMD and TYP on adaptive behavior

Differences between the total SMD group $(n=39)$ and the TYP children $(n=38)$ for adaptive behavior were examined using mixed effects linear regression. As a total group, children with SMD demonstrated significantly lower scores on the communication, daily living, and Adaptive Behavior Composite (ABC) scores than TYP children (Table 6). Next, we examined differences in the SMD latent class groups as compared to TYP. Children in the severe SMD group had significantly lower scores on the communication $(M=93.78$; $p=0.02)$, Daily Living $(M=86.09, p=0.001)$, and ABC $(M=88.32$, $p=0.004)$ compared to TYP children, while the moderate SMD group had lower scores on the Daily Living $(M=89.54, p=0.017)$, Socialization $(M=87.74, p=0.016)$, and ABC $(M=88.64, p=0.009)$. The borderline SMD group was not significantly different than TYP children on any of the adaptive behavior measures.

\section{DISCUSSION \\ DIFFERENCES IN BASELINE VAGAL TONE BETWEEN SMD AND TYP SAMPLES}

As hypothesized, our sample of children with SMD demonstrated lower baseline VT tone that approached significance. in the hypothesized direction. The literature shows that low resting VT is associated with poor developmental outcomes and poor behavioral regulation;

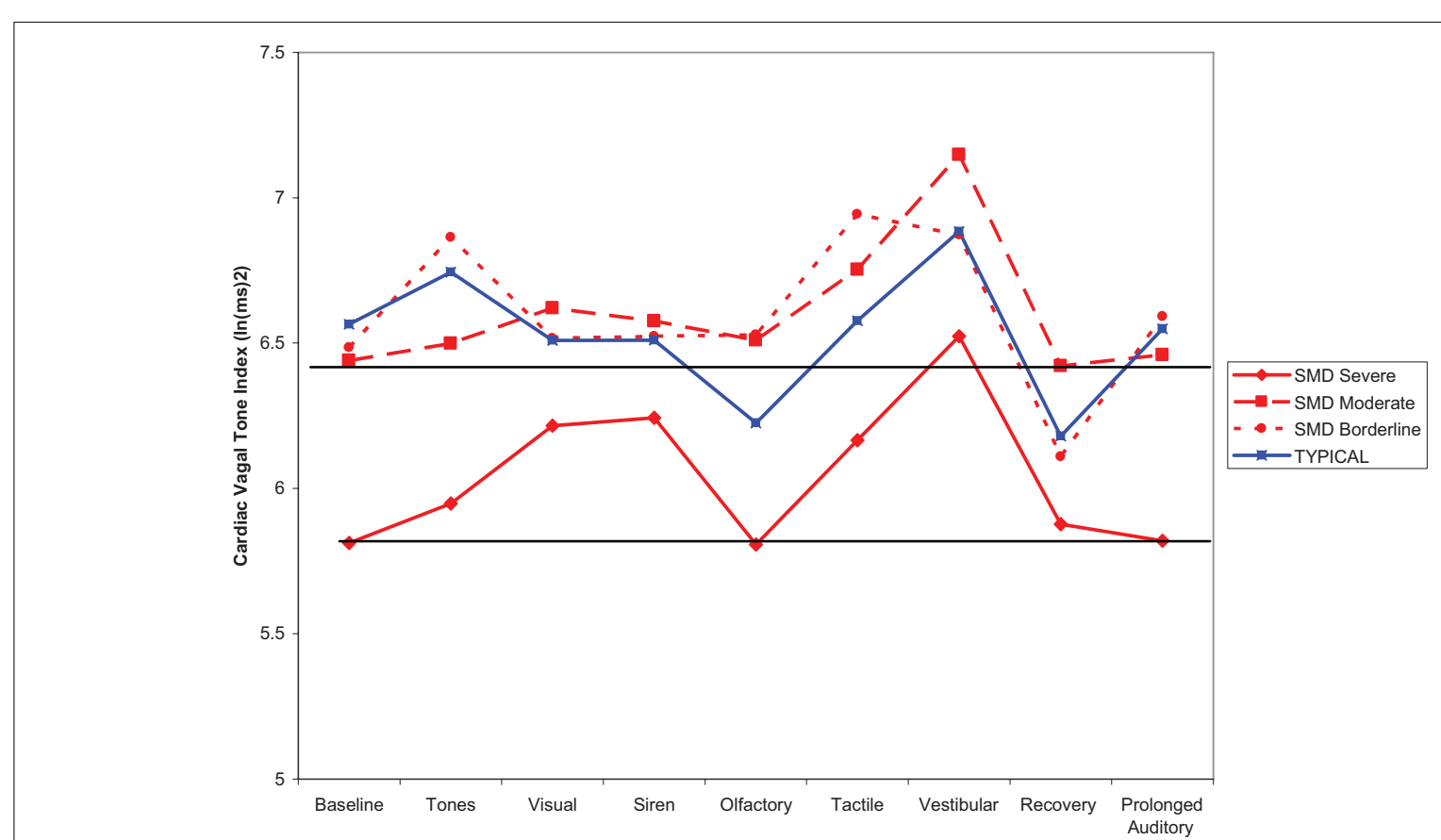

FIGURE 2 |Vagal tone for TYP in comparison to three classes of SMD. 
Table 5 | Mean change in VT scores for SMD subgroups and TYP groups $(95 \% \mathrm{Cl})$.

\begin{tabular}{llccr}
\hline $\begin{array}{l}\text { Change from BL } \\
\text { to SCP domain }\end{array}$ & $\begin{array}{l}\text { SMD group 1, severe; } \\
\text { mean VT change (95\% Cl) }\end{array}$ & $\begin{array}{l}\text { SMD group 2, moderate; } \\
\text { mean VT change (95\% Cl) }\end{array}$ & $\begin{array}{l}\text { SMD group 3, borderline; } \\
\text { mean VT change (95\% Cl) }\end{array}$ & $\begin{array}{l}\text { TYP; mean VT } \\
\text { change (95\% Cl) }\end{array}$ \\
\hline BL to tones & $0.14(-0.19$ to 0.46$)$ & $0.06(-0.03$ to 0.38$)$ & $0.38^{*}(0.00-0.76)$ & $0.18(-0.01$ to 0.36$)$ \\
BL to visual & $0.40^{* *}(0.09-0.72)$ & $0.18(-0.14$ to 0.50$)$ & $0.03(-0.35$ to 0.04$)$ & $-0.06(-0.24$ to 0.13$)$ \\
BL to siren & $0.43^{* *}(0.12-0.74)$ & $0.14(-0.19$ to 0.46$)$ & $0.04(-0.34$ to 0.42$)$ & $-0.05(-0.24$ to 0.13$)$ \\
BL to olfactory & $-0.01(-0.32$ to 0.31$)$ & $0.07(-0.25$ to 0.39$)$ & $0.04(-0.33$ to 0.42$)$ & $-0.34^{* *}(-0.53$ to -0.15$)$ \\
BL to tactile & $0.35^{*}(0.03-0.67)$ & $0.31(-0.00$ to 0.63$)$ & $0.46^{*}(0.08-0.84)$ & $0.01(-0.17$ to 2.0$)$ \\
BL to vestibular & $0.71^{*}(0.37-1.05)$ & $0.71(0.39-1.0)$ & $0.39^{*}(0.01-0.77)$ & $-0.32^{*}(0.13-0.50)$ \\
BL to recovery & $0.064(-0.26$ to 0.39$)$ & $-0.02(-0.34$ to 0.30$)$ & $-0.37^{*}(-0.75$ to 0.00$)$ & $-0.39^{*}(-0.57$ to 1.2$)$ \\
BL to ProAud & $0.01(-0.31$ to 0.33$)$ & $0.02(-0.31$ to 0.35$)$ & $0.11(0.28-0.50)$ & $-0.02(-0.23$ to 0.19$)$ \\
\hline
\end{tabular}

$\mathrm{Cl}$, confidence interval; SCP, Sensory Challenge Protocol; BL, baseline; ProAud, prolonged auditory stimuli.

Change between $B L$ and Stimuli at the ${ }^{*} p<0.05$ level, ${ }^{* *} p<0.01$ level.

Table 6 | Vineland Standard Score differences between SMD and typical.

\begin{tabular}{lccc}
\hline & Mean SMD (95\% Cl) & Mean TYP (95\% Cl) & Estimate (95\% Cl) \\
\hline Communication & $97.02(92.26-101.78)$ & $105.18(99.07-111.28)$ & $-8.16(-16.12$ to -0.20$)$ \\
Daily living & $91.39(86.63-96.16)$ & $102.23(96.12-108.33)$ & $-10.83(-18.79$ to -2.88$)$ \\
Socialization & $93.27(88.51-98.03)$ & $100.56(94.45-106.66)$ & $-7.29(-15.25$ to 0.67$)$ \\
Motor skills & $92.02(86.53-97.52)$ & $99.48(88.55-110.41)$ & $-7.46(-19.81$ to 4.89$)$ \\
ABC & $91.43(86.66-96.19)$ & $102.47(96.30-108.65)$ & $-11.05(-19.06$ to -3.03$)$
\end{tabular}

${ }^{*} p<0.05,{ }^{* *} p<0.01$

whereas high resting (i.e., baseline) VT is associated with better regulatory behaviors (Stifter et al., 1989) and more positive temperament (Calkins, 1997). Our data suggest this pattern also may exist for children with SMD who have poor regulation to sensory stimuli.

When the SMD subjects were grouped by severity, it was the severe SMD group who had significantly lower VT at baseline, tones and prolonged auditory. Given that high resting VT has been associated with better regulation of behavior, the finding of low baseline VT in the children with severe SMD may suggest poor regulation of behavior, specifically as related to sensory stimuli. Extending this finding to intervention, strategies that (1) enhance PsNS activity at rest, and/or (2) enhance one's use of the PsNS to cope with stimuli may be helpful for children with SMD especially those with severe SMD.

Further, it is interesting to note that as a total group the SMD subjects returned to baseline level VT after an overall increase in VT during the SCP; whereas the TYP subject's VT fell below baseline during the recovery period. This finding is interesting in light of the literature about the utility of vagal withdrawal (a decrease in VT after a challenge) suggesting that it may be an indicator of physiological regulation (Calkins et al., 2007). Thus, one possible interpretation is that the SMD group, overall, is not using a robust vagal withdrawal in comparison to the TYP and thus, less physiological regulation.

\section{SENSORY SPECIFIC VAGAL REACTIVITY IN CHILDREN WITH SMD COMPARED TO TYP SAMPLE}

Vagal reactivity is used in the literature to assess the dynamic reactivity of the vagal system. Porges et al. (1996) and Porges (2007) found that suppression of VT during a stressor may function as an adaptive response to increase the individual's orientation to external stimuli thereby allowing regulation and coordination of a more complex response to stimuli. Similarly, Calkins and Keane (2004) showed that specific patterns of vagal change from baseline to stimuli are associated with different temperament and coping styles. In comparing the total group of SMD subjects to the TYP subjects (Figure 1), we note that the TYP subjects' showed minimal reactivity from baseline to stimuli with change scores close to 0 (exception being significant negative change from BL to olfactory and significant positive change from BL to vestibular), whereas the SMD subjects increased VT in response to the stimuli (all stimuli except tones and olfactory show a significant increase from baseline to stimuli). It appears that there are sensory-specific differences between the groups in their parasympathetic activity (and by inference their regulation) during visual, auditory/siren, olfactory, tactile, vestibular and prolonged auditory stimuli. It is possible that the TYP children did not find these stimuli challenging but rather find them interesting therefore, do not display significant changes in vagal reactivity. In contrast, the SMD subjects, rather than withdraw VT as expected, seem to use a parasympathetically mediated response. This is consistent with Katz (2007) whose data suggests that vagal augmentation (an increase in VT) might be reflective of hypervigilance in children with conduct problems who are living in domestically violent homes. Similarly, children with SMD may demonstrate vagal augmentation as a pre-emptive reactionary protective mechanism in attempt to cope with stimuli. (Katz, 2007; Arora et al., 2008; ANSAR Medical Technologies, Inc, Personal conversation, March 27, 2009). In either case, of note, is that the SMD subjects use a different pattern than the TYP children and thus, the children with SMD may be 
processing stimuli differently, using different coping mechanisms, and/or using their autonomic nervous system in a way that is different than TYP children. This finding was consistent even when the SMD subjects were divided into three groups using the latent class analysis. To fully explore differences in autonomic nervous system regulation, additional measures are needed to obtain an appreciation for the complex interactions that occur between parasympathetic and sympathetic systems (Berntson et al., 1991). Analysis of PsNS data combined with sympathetic nervous system data will provide insight into the patterns of autonomic activity that are utilized by children with SMD.

Finally, it is interesting to note that both groups showed a return to baseline during the prolonged auditory condition. This finding suggests that, over time ( $2 \mathrm{~min}$ ) both groups may be habituating to or coping with longer term auditory stimuli. It would be interesting to examine each 30-s epoch of VT during the 2-min prolonged auditory stimuli to determine the patterns for each group and to evaluate any difference in these patterns between the groups (i.e., are there increases or decreases in VT before the return to baseline and are these patterns difference between the group). This idea will be explored in future studies.

\section{SUBGROUPS OF CHILDREN WITH SMD}

Our data suggests that, similar to Miller (2006), there are subgroups of SMD and that these may demonstrate different underlying physiology. However, in contrast to Miller's proposed typology (Miller et al., 2007), our data suggests that the most useful sub grouping strategy is to group by severity of symptoms, rather than by type of symptoms. Given that our sample size was very similar to Miller et al.'s, this finding requires replication with additional subject numbers to determine if this trend continues. The implications of this finding are that children with severe SMD, (classified as having -4 standard deviations below the mean on the SSP in multiple domains), have lowest PsNS regulation, the poorest adaptive behavior, and may require the most intensive and immediate intervention. Children in the severe SMD group were more likely to demonstrate lower VT at baseline, tones, and prolonged tones on the SCP and are likely to demonstrate the poorest physiological regulation.

\section{ADAPTIVE BEHAVIOR IN SMD}

Of interest, the SMD group scored significantly lower than the TYP on two out of the four subdomains of adaptive behavior (communication and daily living subdomains) and the overall ABC, although scores were still within the normal ranges. This finding suggests that children with SMD, are performing below their peers in adaptive behavior skills but may fall above the cut point that qualifies them for services. Hence, children with SMD may not keep pace with their TYP peers in communication and daily living areas, but these may go unrecognized and untreated. It is important, therefore, to educate parents, teachers, psychologists and other professionals who work with these children about the potential impact of their sensory processing on adaptive behavior.

\section{PHYSIOLOGICAL-BEHAVIOR INTERACTIONS}

In regard to VT and SSP scores, it is interesting to note that the severe SMD group had significantly lower baseline VT and the poorest behavioral responses to sensation, suggesting a relationship between physiology (parasympathetic activity at rest) and behavior (sensory responses). Given that the literature shows that high baseline VT is associated with better regulation of behavior, greater social competence, and teacher and parent reports of sociability and emotional regulation (Calkins and Fox, 1992; Eisenberg et al., 1995), this finding is consistent.

\section{LIMITATIONS}

There are several limitations that influence the findings of this study. First, the number of subjects in the subgroup analyses is small and thus the findings need to be replicated with a larger number of subjects. In addition, although we controlled for age in our analysis, we did not control for gender and this may influence results. Second, it is possible that physiological arousal was influenced by the time of day that the subjects were tested, however, we were unable to find any literature that suggests this would impact VT, specifically, and therefore, we did not control for this variable. It should be noted that the vestibular stimuli during The SCP uses a motorized chair to deliver the vestibular stimulus. The quality of the stimulus is confounded by sound and vibration from the motor. Since the movement stimuli is not solely vestibular and not felt to be a pure measure of vestibular reactivity the vestibular data may reflect multisensory reactivity rather than pure vestibular reactivity. In addition, this study utilized a convenience sample of children with SMD. While some children had been referred by clinicians, many children were self-referred by parents who were interested in knowing more about their child's sensory responses and thus the SMD sample may be biased. A small percentage of the children had a co-morbid diagnoses (i.e., apraxia, learning disorders) but also met the inclusion criteria for SMD and thus were included in the sample. Of note, we did not control for IQ scores as the literature suggests that sensory modulation is independent of IQ (Ashburner et al., 2008; Chen et al., 2008). Finally, the findings from this study would be enhanced by using additional measures of autonomic nervous system activity, specifically measures of sympathetic activity that could be compared to PsNS responses to yield a more insight into autonomic balance and flexibility in these groups.

\section{CONCLUSION}

Despite the caveats listed above, this study presents some new and useful information about children with SMD. This is one of the first studies to examine PsNS functioning in children with SMD to determine if it is a useful biomarker of sensory dysfunction. Our main finding is that children with the most severe SMD demonstrate significantly lower baseline VT, and lower VT during auditory (tones and prolonged tone) sensory challenges. Future research should focus on quantifying the severity of sensory dysfunction, both from a physiological and behavioral perspective. Clinically, it is possible that the children who demonstrate the most severe sensory behaviors have the most notable physiological dysregulation. Continued research to determine the impact of SPDs and autonomic dysregulation can support future intervention studies utilizing measures of autonomic responses to determine if treatment improves physiological regulation and thus, behavior. 


\section{ACKNOWLEDGMENTS}

The authors gratefully acknowledge the Wallace Foundation who provided support for this study, and the parents and children who volunteered their time to participate in this study in the Philadelphia, PA and Torrance, CA regions. In addition, the authors gratefully acknowledge assistance in data collection by

\section{REFERENCES}

Ahn, R. R., Miller, L. J., Milberger, S., and McIntosh, D. N. (2004). Prevalence of parents' perceptions of sensory processing disorders among kindergarten children. Am. J. Occup. Ther. 58, 287-293.

Arora, R. R., Ghosh-Dastidar, S., and Colombo, J. (2008). Altered sympathetic and parasympathetic activity is associated in patients with chronic coronary artery disease. Clin. Auton. Res. 18, 276 (abstract).

Ashburner, J., Zivani, J., and Rogers, S. (2008). Sensory processing and classroom emotional, behavioral and educational outcomes in children with autism spectrum disorder. Am. J. Occup. Ther. 62, 564-573.

Ayres, A. J. (1969). Deficits in sensory integration in educationally handicapped children. J. Learn Disabil. 2, 160-168.

Ayres, A. J. (1972). Sensory Integration and Learning Disorders. Los Angeles, CA, Western Psychological Services.

Ayres, A. J. (1979). Sensory Integration and the Child. Los Angeles, CA, Western Psychological Services.

Ayres, A. J.(1989). The Sensory Integration and Praxis Tests. Los Angeles, CA, Western Psychological Services.

Baranek, G. T. (1998). Sensory processing in persons with autism and developmental disabilities: considerations for research and clinical practice. Sens. Integr. Spec. Interest Sect. Q. 21, 1-3.

Beauchaine, T. (2001). Vagal tone, development, and gray's motivational theory: toward an integrated model of autonomic nervous system functioning in psychopathology. Dev. Psychopathol. 13, 183-214.

Berntson, G. G., Bigger, J. T., Eckberg, D. L., Grossman, P., Kaufman, P. G., Malik, M., Nagaraja, H. N., Porges, S. W., Saul, J. P., Stone, P. H., and Van Der Mole, M. W. (1997). Heart rate variability: origins, methods, and interpretive caveats. Psychophysiology 34, 623-648.

Berntson, G. G., Cacioppo, J. T., and Quigley, K. S. (1991). Autonomic determinism: the modes of autonomic control, the doctrine of autonomic space and the laws of autonomic constraint. Psychol. Rev. 98, 459-487.

Berntson, G. G., Norman, G. J., Hawkley, L. C., and Cacioppo, J. T. (2008). Cardiac autonomic balance versus cardiac regulator capacity. Psychophysiology $45,643-652$.

Boccia, M. L., and Roberts, J. E. (2000). Behavior and autonomic nervous system function assessed via heart period measures: the case of hyperarousal in boys with fragile X syndrome. Behav. Res. Methods Instrum. Comput. 32, 5-10.

Bundy, A. C., Lane, S. J., and Murray, E. A. (2002). Sensory Integration: Theory and Practice, 2nd Edn. Philadelphia, PA, F.A. Davis.

Cacioppo, J. T., Uchino, B. N., and Berntson, G. G. (1994). Origins of baseline variance and the law of initial values. Psychophysiology 31, 204.

Calkins, S., and Fox, N. A. (1992). The relations among infant temperament, security of attachment and behavioral inhibition at twenty-four months. Child Dev. 63, 1456-1472.

Calkins, S., Graziano, P. A., and Keane, S. P. (2007). Cardiac vagal regulation differentiates among children at risk for behavior problems. Biol. Psychol. $75,144-153$.

Calkins, S. D. (1997). Cardiac vagal tone indices of temperamental reactivity and behavioral regulation in young children. Dev. Psychobiol. 31, 125-135.

Calkins, S. D., and Keane, S. P. (2004). Cardiac vagal regulation across the preschool period: Stability, continuity, and implications for childhood adjustment. Dev. Psychobiol. 45, 101-112.

Chambers, A. S., and Allen, J. B. (2007). Cardiac vagal control, emotion, psychopathology, and health. Biol. Psychol. 74, 113-115.

Chen, Y., Rodgers, J., and McConachie, P. (2008). Restricted and repetitive behaviours, sensory processing and cognitive style in children with autism spectrum disorders. J. Autism Dev Disord. 39, 635-642.

Contact Precision Instruments. (2002). PSYLAB Sam Physiological Data Logger. London, Contact Precision Instruments.

DeGangi, G.A., DiPietro, J.A., Greenspan, S. I., and Porges, S. W. (1991) Psychophysiological characteristics of the regulatory disordered infant. Infant Behav. Dev. 14, 37-50.

Delta Biometrics. (1993). MXEdit. Bethesda, MD, Delta Biometrics.

the graduate research assistants at Thomas Jefferson University. The authors also thank the collaborating researchers and clinicians at Pediatric Therapy Network in Torrance, CA, for their time and effort in recruitment of families and support of this study, including Gina Coleman, Annie Baltazar, OTD, OTR/L, and Karla Medellin.

DiPietro, J. A., Larson, S. K., and Porges, S.W. (1987). Behavioral and heart rate pattern differences between breast-fed and bottle-fed neonates. Dev. Psychol $23,467-474$.

Dunn, W. (1999). The Sensory Profile: User's Manual. San Antonio, TX Psychological Cooperation.

Eisenberg, N., Fabes, R. A., and Murphy, B. C. (1995). Relations of shyness and low sociability to regulation and emotionality. J. Pers. Soc. Psychol. 68 505-517.

Fox, N. A., and Porges, S. W. (1985). The relation between neonatal heart period patterns and developmental outcome. Child Dev. 56, 28-37.

Fox, N. A., and Stifter, C. A. (1989). Biological and behavioral differences in infant reactivity and regulation. In Temperament in Childhood, G. A. Kohnstamm, J. E. Bates and M. K. Rothbart, eds. (New York, Wiley), pp. 169-183.

Hollingshead,T.S.(1991).Pathophysiology and treatment of diabetic foot ulcer Clin. Podiatr. Med. Surg. 8, 843-855.

Huffman, L. C., Bryan, Y. E., del Carmen, R., Pedersen, F.A., Doussard-Roosevelt, J. A., and Porges, S. W. (1998). Infant temperament and cardiac vagal tone: assessments at twelve weeks of age Child Dev. 69, 624-635.

Katz, L. F. (2007). Domestic violence and vagal reactivity to peer provocation. Biol. Psychol. 74, 154-164.

Lane, S. J. (2002). Sensory modulation. In Sensory Integration: Theory and Practice, 2nd Edn, A. C. Bundy, S. J. Lane and E. A. Murray, eds (Philadelphia, F.A. Davis).

Malliani, A. (1995). Association of heart rate variability components with physiological regulatory mechanisms. In Heart rate Variability, M. Malik and A. J. Camm, eds (Armonk, NY, Futura Publishing Company Inc.).

McIntosh, D. N., Miller, L. J., Shyu, V., and Dunn, W. (1999a). Overview of the short sensory profile. In The Sensory Profile: Examiner's Manual,W.Dunn, ed. (San Antonio, The Psychological Corporation), pp. 59-73.

McIntosh, D. N., Miller, L. J., Shyu, V., and Hagerman, R. J. (1999b). Sensory-modulation disruption, electrodermal responses, and functional behaviors. Dev. Med. Child Neurol. 41, 608-615.
Miller, L. J. (2003). Empirical evidence related to therapies for sensory processing impairments. NASP Commun. 31, 34-37.

Miller, L. J. (2006). Sensational Kids: Hope and Help for Children With Sensory Processing Disorders. New York, Putnam Press.

Miller, L. J., Anzalone, M. E., Lane, S. J. Cermak, S. A., and Osten, E. T. (2007). Concept evolution in sensory integration: A proposed nosology for diagnosis. Am. J. Occup. Ther. 61, 135-141.

Miller, L. J., McIntosh, D. N., McGrath, J., Shyu, V., Lampe, M., Taylor, A. K., Tassone, F., Neitzel, K., Stackhouse, T., and Hagerman, R. (1999). Electrodermal responses to sensory stimuli in individuals with fragile X syndrome. Am. J. Med. Genet. 83, 268-279.

Miller, L. J., Reisman, J. E., McIntosh D. N., and Simon, J. (2001). An ecological model of sensory modulation: performance of children with fragile $\mathrm{X}$ syndrome, autistic disorder, attentiondeficit/hyperactivity disorder, and sensory modulation dysfunction. In Understanding the Nature of Sensory Integration With Diverse Populations, S. Smith Roley, E. I. Blanche and R. C. Schaaf, eds (San Antonio, TX, The Psychological Corporation), pp. 57-88.

Miller, L. J., Schoen, S. A., and BrettGreen, B. (2003). Physiological and Behavioral Responses to Sensation in Children With Autism Spectrum Disorders. Final Report. Los Angeles, CA, Cure Autism Now Foundation (unpublished).

Miller, L. J., Shyu, V., and Dunn, W. (1999). The short sensory profile. In The Sensory Profile, W. Dunn, ed. (Tucson, Psychological Corporation).

Nance, P. W., and Hoy, C. S. (1996). Assessment of the autonomic nervous system. Phys. Med. Rehabil. 10, 15-35.

Porges, S. W. (1980). Individual differences in attention: a possible physiological substrate. Adv. Spec. Educ. 2, 111-134.

Porges, S. W. (1981). Vagal mediation of respiratory sinus arrhythmia. Ann. N. Y. Acad. Sci. 618, 57-66.

Porges, S. W. (1985). Method and Apparatus for Evaluating Rhythmic Oscillations in Aperiodic Physiological 
Response Systems. US Patent No. 4 520944.

Porges, S. W. (1995). Cardiac vagal tone: a physiological index of stress. Neurosci. Biobehav. Rev. 19, 225-233.

Porges, S. W. (2007). The polyvagal perspective. Biol. Psychol. 74, 116-143.

Porges, S. W., and Byrne, E. A. (1992). Research methods for measurement of heart rate and respiration. Biol. Psychol. 34, 93-130.

Porges, S. W., Doussard-Roosevelt, J. A., Portales,A.L., and Greenspan, S.I.(1996). Infant regulation of the vagal "brake" predicts child behavior problems: a psychobiological model of social behavior. Dev. Psychobiol. 29, 697-712.

Schaaf, R. C., Miller, L. J., Seawell, D., and O'Keefe, S. (2003). Children with disturbances in sensory processing: a pilot study examining the role of the parasympathetic nervous system. Am. J. Occup. Ther. 57, 442-449.

Sparrow, S. S., Cicchetti, D. V., and Balla, D. A. (2005). The Vineland Adaptive Behavior Scales II: Survey Forms Manual, 2nd Edn. Circle Pines, MN, American Guidance Service.

Stifter, C. A., Fox, N. A., and Porges, S. W. (1989). Facial expressivity and vagal tone in 5- and 10-month-old infants. Infant Behav. Dev. 12, 127.

Conflict of Interest Statement: The authors declare that the research was conducted in the absence of any commercial or financial relationships that could be construed as a potential conflict of interest.

Received:01 April 2009; paperpending published: 11 August 2009; accepted:09 February 2010; published online: 09 March 2010. Citation: Schaaf RC, Benevides T, Blanche EI, Brett-Green BA, Burke JP, Cohn ES, Koomar J, Lane SJ, Miller LJ, May-Benson
TA, Parham D, Reynolds $S$ and Schoen SA (2010) Parasympathetic functions in children with sensory processing disorder. Front. Integr. Neurosci. 4:4. doi: 10.3389/fnint.2010.00004

Copyright (c) 2010 Schaaf, Benevides, Blanche, Brett-Green, Burke, Cohn, Koomar, Lane, Miller, May-Benson, Parham, Reynolds and Schoen. This is an open-access article subject to an exclusive license agreement between the authors and the Frontiers Research Foundation, which permits unrestricted use, distribution, and reproduction in any medium, provided the original authors and source are credited. 